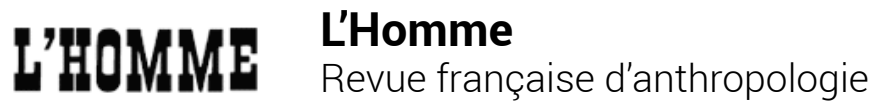

173 | 2005

Varia

\section{Pour une anthropologie des pratiques historiographiques}

\section{Claude Calame}

\section{(2) OpenEdition}

\section{Journals}

\section{Édition électronique}

URL : http://journals.openedition.org/lhomme/25015

DOI : 10.4000/lhomme.25015

ISSN : 1953-8103

\section{Éditeur}

Éditions de l'EHESS

\section{Édition imprimée}

Date de publication : 1 mars 2005

Pagination : 11-45

ISSN : 0439-4216

\section{Référence électronique}

Claude Calame, "Pour une anthropologie des pratiques historiographiques », L'Homme [En ligne],

173 | 2005, mis en ligne le 01 janvier 2007, consulté le 19 avril 2019. URL : http://

journals.openedition.org/lhomme/25015; DOI : 10.4000/lhomme.25015 


\title{
Pour une anthropologie des pratiques historiographiques
}

\author{
Claude Calame
}

C

ONSCIENTES de leurs fondements narratifs et poétiques, les pratiques historiographiques s'inscrivent désormais dans une tension entre sollicitations du présent et poids de la tradition, avec son exigence de mémoire. C'est en particulier à ce titre que les historiens sont désormais invités à esquisser et formuler des réponses critiques à des interrogations et à des exigences actuelles quant au passé de leurs communautés.

Dans un tel contexte, la célèbre conception philosophique du temps esquissée par saint Augustin peut prendre sens aux yeux de qui s'estime concerné par des conceptions pratiques de la temporalité, envisagées dans une perspective anthropologique :

"Qu'est-ce en effet que le temps (quid est enim tempus?)? [...] Ces deux temps que sont le passé et le futur comment existent-ils quand le passé déjà n'est plus et que le futur n'est pas encore? Quant au présent, s'il était toujours présent et qu'il ne se changeait pas en passé, il ne serait plus le temps, mais l'éternité».

Même philosophiquement, le présent ne peut se concevoir si ce n'est comme tension entre le passé et le futur. Pour donner une consistance au

Encore partielles, les réflexions proposées ici ont guidé l'exposé de quatre régimes et logiques de temporalité et de spatialité hellènes, prononcé dans le cadre des Carl Newell Jackson Lectures données à l'Université de Harvard en octobre 2000 ; elles ont également fondé l'animation du séminaire donné à l'École des hautes études en sciences sociales en 2001-2002, ainsi que différentes interventions dans l'atelier franco-hongrois de l'Université Eötvös Lorand à Budapest, à l'occasion d'une journée sur le "Temps multiple" au CAMS (EHESS), dans le Séminaire de morphogenèse de l'EHESS et dans le Séminaire intersémiotique de Paris ("Temps et discours»). Je tiens à remercier les auteurs des observations et interrogations que ces propositions ont pu susciter à ces quelques occasions. Interprétées, sans doute à raison, comme la marque d'un narcissisme intellectuel inopportun, les nombreuses références à des travaux personnels précédents ont été imposées par la volonté d'inscrire ces réflexions dans le cours d'une recherche ouverte. Le lecteur voudra bien excuser les redondances qu'une telle intention a pu entraîner. 
présent et donc au temps, il convient de l'envisager de manière tensive à la fois comme souvenir et comme attente ${ }^{1}$.

Mais - faut-il le remarquer d'emblée ? - le moment temporel présent implique également une consistance et un repérage spatiaux. Alors que les physiciens réfléchissent depuis près d'un siècle sur la question de la courbure d'un temps indissociable de l'espace, c'est un tel constat qu'impose ici la dimension anthropologique et discursive de l'histoire défendue dans cet article. Se pose dès lors la question du statut ontologique de cette dynamique du flux temporel axé aussi bien sur le moment que sur l'espace présents. Ainsi, faire de l'écoulement du temps un mouvement de l'animus comme le propose saint Augustin dans une dialectique entre intentio et distentio, c'est en définitive envisager le temps dans une dimension d'ordre psychologique. Tout en esquivant la question de l'articulation de la temporalité intérieure avec le temps social ou avec le temps cosmique, cette conception gomme les paramètres spatiaux de toute temporalité. On y reviendra.

À cet égard, les textes issus des manifestations culturelles et symboliques de la Grèce ancienne offrent des représentations du temps et de l'espace significatives. Elles sont d'autant plus intéressantes que, portées par des textes poétiques à fonction pratique, elles sont toujours suscitées et conditionnées, comme les nôtres d'ailleurs, par une préoccupation ancrée dans le hic et nunc, en relation avec le passé. En général orientées vers une action de l'ordre du rituel accompli en un lieu précis, ces manifestations spatio-temporelles entretiennent par ailleurs une relation pragmatique avec le futur immédiat. Il s'agit donc de sentiments, mais aussi de représentations et finalement, en raison de la mise en discours dont ces représentations sont l'objet, de configurations de la temporalité et de la spatialité; dans leur aspect pratique, elles se distinguent fortement des conceptions philosophiques du temps que l'on a trop volontiers attribuées aux représentants de la culture grecque en général, faisant de poètes des métaphysiciens. Représentation, configuration, mise en discours, trois notions dont on aura à préciser la définition, et qui peuvent animer, à travers les conceptions pragmatiques proposées par la culture hellène, la réflexion sur nos propres pratiques historiennes. D'où la double dimension de l'essai sur une anthropologie de l'histoire présenté ici : approche de quelques modes hellènes de faire l'histoire pour réfléchir sur nos propres manières de constituer notre passé en histoire. saint Augustin dont il montre les implications théologiques et les insuffisances pratiques. 


\section{Pour une pragmatique des représentations spatio-temporelles}

On a trop souvent ignoré que les conceptions grecques du temps et de l'espace se situent à l'entrecroisement de plusieurs des lignes temporelles qui apparaissent comme constitutives de notre être en société. À titre opératoire et de manière par conséquent schématique, il faut rappeler que l'on peut distinguer plusieurs lignes de réalisation dans nos sentiments et nos expressions d'une temporalité (et d'une spatialité) que l'on considérera globalement comme existentielle. Je reprendrai volontiers et naïvement ici la distinction traditionnellement tracée entre temps cosmique et temps vécu. Le premier correspond au temps et à l'espace physiques, à cet espacetemps universel qui entraîne le monde dans une dynamique de modifications matérielles et organiques apparemment inexorable, par l'effet probable d'un principe d'entropie qui en définit l'irréversibilité (cf. Paty 1996 : 52-55) ; le second, souvent envisagé aussi comme temps psychique ou temps ordinaire, coïncide avec la perception que chaque humain a du temps physique et avec l'expérience qu'il en fait dans son corps propre et dans son esprit. En concomitance, on pourra d'ailleurs tracer une distinction complémentaire et également opératoire entre un espace "naturel " ou organique et un espace perçu et finalement construit.

\section{Temporalités philosophiques}

Or, c'est précisément dans les pratiques de l'histoire qu'un philosophe tel que Paul Ricœur espère trouver les points permettant de surmonter les apories d'une temporalité partagée entre temps «objectif» et temps «phénoménologique ", ou, pour parler dans les termes d'une phénoménologie heideggerienne à nouveau en vogue, c'est dans le domaine de l'histoire que seraient à chercher les passerelles entre un temps extérieur et mesurable, et un temps intérieur, le temps du Dasein. Au nom du statut ontologique du second, le premier serait à rejeter, dans un paradoxe pour le moins significatif, en tant que temps "vulgaire », un temps dénommé ainsi parce qu’il serait noyé dans une succession d'événements aussi anecdotiques qu'aléatoires... Dès lors, le temps du Dasein se trouverait marqué par une tension vers le futur. En dépit de son statut d'ordre ontologique, une telle tension pourrait être stimulante si elle ne se révélait animée par un souci existentiel et métaphysique pour le moins malsain, et si elle ne finissait pas par coïncider, sur un mode décidément morbide, avec «l'être-pour-la-mort » ${ }^{2}$.

2. Cf. Ricœur (1985: 147-182), tentant de résoudre l'aporie à laquelle se trouve confronté, dans son rejet méprisant du temps quotidien, Heidegger (1986 [1927] : 472-506 [407-437] ); à propos de ces pages inquiétantes, voir encore Ricœur (1983: 125-129), et (1985: 90-144). 
Grâce aux artifices de langage naguère dénoncés par Pierre Bourdieu, puis par Henri Meschonnic, et dans une formulation où les habituelles redondances étymologisantes sont soulignées par les astuces typographiques d'une traduction absurdement mimétique, Martin Heidegger peut par exemple affirmer :

«Jeté en déval, le Dasein est d'abord et le plus souvent perdu dans son occupation. Mais dans cet être-perdu s'annonce la fuite par laquelle le Dasein se dissimule son existence propre, celle qui a été caractérisée comme résolution en marche. Qui dit fuite dans la préoccupation, dit fuite devant la mort, c'est-à-dire une façon de dé-tourner la vue de la fin de l'être-au-monde. Cette façon de détourner le regard de... est en ellemême un mode de l'être vers la fin ekstatiquement tendu vers l'avenir. Du moment qu'elle détourne ainsi sa vue de la finitude, la temporellité impropre du Dasein dans son dévalement quotidien doit méconnaître l'être à venir propre et donc aussi la temporellité en général »³.

En fait, si «être-pour-la-mort» il y a pour chaque individu - on sait qu'Hésiode lui-même ne l'aurait pas contesté... -, tout Dasein dépend d'un temps vécu, "extérieur", qui est lui-même fondé dans le flux de l'espacetemps physique et cosmique. (De ce point de vue, c'est un complet renversement de perspective que j'entends proposer ici, en plaidant pour une conception anthropologique et pragmatique de l'histoire.)

L'aporie à laquelle conduit un temps ontologique et philosophique orienté vers la mort au mépris des possibilités de réalisation et de construction du temps social et pratique à travers l'histoire est donc évidente. Face à cette impasse philosophique, on réexaminera volontiers la double tentative proposée par Paul Ricœur de redonner, d'une part, à l'histoire une fonction de "réinscription du temps vécu sur le temps cosmique", et, d'autre part, de fonder la condition historique de l'homme, avec son éventuelle herméneutique, dans la dialectique de la mémoire et de l'oubli. La tentative la plus ancienne est fondée - rappelons-le - sur une représentation modélisante de la construction du temps historique articulée en trois phases, dans un enchaînement de processus dont le statut cognitif n'est d'ailleurs pas précisé :

- opérations de "préfiguration" dans une compréhension à la fois pratique et symbolique de l'action humaine et de sa temporalité (mimèsis I) ;

- processus de "configuration" par la mise en intrigue, d'ordre narratif et discursif, des actions de l'homme organisées en une séquence temporelle (mimèsis II) ;

3. Heidegger 1986 [1927] : 492 [425]. Le mépris du social et de la pratique impliqué par de telles formulations sophistiques a été dénoncé avec beaucoup de pertinence par Bourdieu 1982: 167205 [2001: 343-377]; sur les effets trompeurs de la rhétorique heideggerienne, voir Meschonnic (1990 : 346-373) notamment. 
- «refiguration» du temps configuré par les moyens de la narration quand ce temps est en quelque sorte rendu à l'expérience humaine (mimèsis III).

Le projet s'inscrit dans la définition d'une herméneutique qui, par l'attention portée à l'analyse sémio-narrative qui s'est développée dans les années 1970, reprend à son compte la structure tripartite et les procédures impliquées par le schéma de la communication appliqué à la production littéraire.

"Une herméneutique, en revanche, est soucieuse de reconstruire l'arc entier des opérations par lesquelles l'expérience pratique se donne des œuvres, des auteurs et des lecteurs [...]. L'enjeu est donc le procès concret par lequel la configuration textuelle fait médiation entre la préfiguration du champ pratique et sa refiguration par la réception de l'œuvre [...]. Aristote, nous l'avons vu, a ignoré les aspects temporels de la mise en intrigue. Je me propose de les désimpliquer de l'acte de configuration textuelle et de montrer le rôle médiateur de ce temps de la mise en intrigue entre les aspects temporels préfigurés dans le champ pratique et la refiguration de notre expérience temporelle par ce temps construit. Nous suivons donc le destin d'un temps préfiguré à un temps refiguré par la médiation d'un temps configuré»".

Préfiguration, configuration, refiguration : trois notions que l'on retiendra volontiers ici, mais dans une perspective critique quant à leur application.

En revanche, dans l'étape la plus récente de son long parcours, Paul Ricour s'est enfin aperçu qu'en définissant les travaux des historiens comme des configurations ou plutôt des refigurations d'ordre discursif et plus particulièrement narratif de différentes préfigurations du temps, il se trouvait de fait confronté à l'épineux problème de la référence linguistique. Il est évident que si l'on conçoit le problème général de l'articulation entre temps phénoménologique et temps cosmique en termes de mise en intrigue, sinon de mise en discours (historique...), se pose alors la question de savoir par quelles médiations la "réalité" spatio-temporelle du passé, les événements, sont constitués en histoire. Au-delà du rôle de préfiguration joué par l'indispensable mémoire individuelle, au-delà de la matérialisation des fragments de mémoires singulières et collectives en archives, la simple mise en intrigue de type narratif se révèle à l'évidence insuffisante pour rendre compte des opérations intellectuelles relatives à la configuration dans le mouvement de mimèsis II. Ces opérations seraient à regrouper en trois catégories impliquant un ordre de succession : aux opérations d'archivage succéderaient les procédures de l'explication/compréhension, qui déboucheraient quant à elles sur des représentations saisies

4. Ricœur 1983 : 86-87 (1985: 147 pour la première citation). Les trois moments de la mimèsis fondant l'herméneutique sont décrits par Ricœur (1983: 87-100, 101-109 et 109-129 successivement). 
comme interprétations dans la « représentance». Et de corriger la position adoptée dans le premier triptyque :

«Le problème de la référentialité propre au discours historique me paraît se poser de manière distincte, dans la mesure où une tendance à la clôture, inhérente à l'acte de mise en intrigue, fait obstacle à la pulsion extralinguistique, extratextuelle, pour tout dire référentielle, par quoi la représentation se fait représentance " 5 .

On verra qu'il convient d'aller beaucoup plus loin dans la critique portant sur le privilège accordé à la mise en intrigue dans les procédures relatives à l'acte de configuration et de représentation, à l'écart de la question de la référence.

Défini dans le volet initial de la première enquête conduite par Paul Ricœur sur le temps de l'histoire, le processus en trois moments mimétiques s'avère déterminant quand, dans le troisième volet du parcours proposé dans les années 1980, se pose la question du «tiers-temps" susceptible d'assurer la médiation entre le temps cosmique et le temps vécu pour trouver une issue aux impasses de la phénoménologie (heideggerienne) du temps :

«Une troisième option, ouverte par la rumination des apories de la phénoménologie du temps, consiste à réfléchir sur la place du temps historique entre le temps phénoménologique et le temps que la phénoménologie ne réussit pas à constituer, quion l'appelle temps du monde, temps objectif ou temps vulgaire" (Ricour 1985: 147).

On aura compris que le «tiers-temps » recherché coïncide avec l'histoire ; celle-ci opérerait un véritable travail d'ordre poétique à partir d'«instruments de pensée " tels que le calendrier, la succession des générations ou le recours aux archives et documents (Ricour 1985: 153). En l'absence du renversement de perspective proposé ici et en raison du statut ontologique conféré à la temporalité du Dasein, on ne s'étonnera pas de voir Paul Ricœur situer en définitive le travail de l'historien parmi les procédures de la phase de refiguration, c'est-à-dire du côté de la relecture et de l'interprétation. En effet, dans le retour de Paul Ricœur à la question philosophique du temps, la fabrication de l'histoire est assimilée à un processus de lecture et de relecture de configurations temporelles déjà constituées. Le «tiers-temps » censé servir de médiation, par la mise en intrigue, entre le temps quotidien du vécu et le temps physique et organique, sinon cosmique, serait par conséquent déplacé de la mimèsis II à la mimèsis III ; il serait à trouver dans les procédures de la refiguration!

Cette étrange relégation du travail de l'historien, et de l'histoire ellemême, du côté de la refiguration en mimèsis III est sans doute provoquée autant par l'aporie à laquelle conduit la position défendue par Martin 
Heidegger que par la place assignée à l'interprétation par l'herméneutique traditionnelle : d'une part, comprise comme intratemporalité, l'opération historiographique ne serait qu'un dérivé de l'historicité constitutive du Dasein; d'autre part, la perspective herméneutique latente adoptée par Paul Ricœur fait que le moment interprétatif ne peut se situer que dans la refiguration, en aval de l'élaboration poiétique et par conséquent polysémique qu'impliquent les opérations de configuration dans la mise en discours. Or si «tiers-temps » il y a, il n'est pas à chercher dans l'articulation entre temps cosmique et temps vécu, tant il est vrai que le second est immanquablement imbriqué et entraîné dans et par le flux inéluctable du premier. C'est sur l'espace-temps cosmique et physique, c'est aussi dans la temporalité organique du corps humain que se fonde en fait et en dernier ressort l'historicité constitutive des hommes, de leurs communautés, de leurs cultures; des communautés et des cultures dont l'histoire est repérée dans l'espace, selon des paramètres géographiques et écologiques souvent passés sous silence. Non pas le temps institué en "temporellité " générale dans l'homme-Dasein, mais l'homme et ses communautés dans l'espacetemps, un espace-temps qu'ils vivent et qu'ils façonnent culturellement, par différentes procédures $\mathrm{d}^{\prime}$ " anthropopoiésis $»^{6} \ldots$

À la faveur de ce renversement de perspective, le concept de «tierstemps " doit être remplacé par celui de «médiations spatio-temporelles »:

- médiations d'une part dans les préfigurations du temps et de l'espace du vécu (ancrées dans le temps et l'espace physiques) avec la mémoire individuelle et collective qui leur correspond ;

- médiations d'autre part dans les différentes opérations de l'histoire qui retravaille ces préfigurations pour les fonder non pas en un temps philosophique, sinon métaphysique, mais en une mémoire communautaire d'ordre pratique ;

- médiations enfin par le fait que les opérations de la mémoire historique que connaît toute communauté culturelle, à travers différents genres oraux et écrits, s'inscrivent à leur tour dans une historicité et dans une spatialité à la fois individuelles et collectives et qu'elles y trouvent, dans des refigurations, une efficacité pratique.

Dans cette perspective, qu'elle soit confiée à des poètes spécialistes de la mémoire de la communauté ou à des universitaires se réclamant de méthodes érudites et académiques, l'histoire en tant que pratique de recréation par différents modes de la mise en discours n'est pas refiguration, mais configuration. Elle est " poiétique » - comme on le verra - au sens étymo-

6. Sur ce concept et ses implications voir les différentes contributions d'Affergan, Borutti, Calame, Fabietti, Kilani \& Remotti 2003. 
logique du terme; comme dans la Grèce des aèdes homériques, elle reste fille de Mnémosyné. Mais en tant que pratique sociale, elle a en retour un impact sur le flux spatial et temporel de notre vécu singulier et communautaire. Par le biais herméneutique - sans doute -, il appartient aux opérations de la refiguration de réaliser cette dimension pragmatique essentielle à toute opération de mise en discours et de configuration historienne. C'est par la refiguration que l'histoire-configuration peut réorienter le flux spatio-temporel qui constitue notre historicité dans l'espace-temps physique. Loin d'être accidentel et "vulgaire», ce flux est le référent des opérations mémoriales de l'histoire comme genre poiétique et comme discipline académique, des opérations qui devraient nous aider à le gérer!

Aussi reconnaitra-t-on volontiers que les effets pratiques et sociaux de la mise en discours qui fonde toute configuration notamment narrative dans le travail de mimèsis II passent par la refiguration et ressortissent par conséquent à mimèsis III. Il en va ainsi, à vrai dire, des retombées pratiques de toute manifestation du processus symbolique envisagé dans sa dimension pragmatique. En revanche, en regard des opérations qu'il est possible d'envisager pour rendre compte de la production symbolique et fictionnelle en général, l'activité de l'historien et ses produits relèvent assurément des procédures de configuration et de mise en intrigue de mimèsis $\mathrm{II}^{7}$. Même si on aura l'occasion d'en définir plus précisément les contours, il convient d'emblée d'indiquer que la notion de configuration recouvre, au-delà de la mise en intrigue, toute une série de procédures de mise en forme discursive; avec la mise en séquence, ces procédés combinent la description, la schématisation, les différentes variétés de la métaphore et une logique d'ordre argumentatif, sinon explicatif; la configuration tend donc à une modélisation visant à rendre intelligible ${ }^{8}$.

Dans cette perspective, la configuration renvoie à la notion plus englobante de la représentation ; cette dernière subsume les différentes manières dont on saisit un état de choses sous une forme figurative et symbolique, c'est-à-dire sous une forme po(i)étique au sens grec et étymologique d'un terme que l'on aura encore à définir. Développée à l'issue de l'ultime étape de l'enquête de Paul Ricœur, la notion de "représentance» ne saurait se substituer à celle de la représentation. Désignant «l'attente attachée à la

7. Par exemple, un historien de l'historiographie comme Alain Prost (1996: 247-252) envisage bien l'" acte fondateur " que constitue la construction de l'intrigue comme un travail de configuration. En 1996b : 15-68, j'ai tenté de montrer les impacts pratiques et sociaux qui sont inhérents à toute mise en discours d'ordre symbolique.

8. Sur quelques-unes de ces opérations de la configuration historiographique, voir en dernier lieu la contribution de Jacques Revel (2001 : 62-74). Ricœur (2000: 320-338) reconnaît désormais l'existence des « ressources rhétoriques de l'attestation verbale», notamment dans les mouvements d'attestation et de protestation offerts par les récits de la Shoah. 
connaissance historique des constructions constituant des reconstructions du cours passé des événements» et fondée sur "l'intentionnalité historienne ", la représentance se situerait à son tour du côté de mimèsis III" Assurément, la faculté de "mettre sous les yeux », conçue comme enargeia par les rhétoriciens de l'Antiquité, est centrale dans l'opération de restitution d'un référent spatio-temporel. Mais elle se réalise dans l'opération de configuration avant de déployer ses effets pragmatiques dans le moment de refiguration, peut-être marqué par un "pacte entre l'écrivain et le lecteur ». Si la conviction est bien provoquée auprès du public par la force d'une écriture littéraire ${ }^{10}$, cela signifie qu'il est impossible de faire un partage net entre opération historienne et œuvre de fiction. Toutes deux sont en définitive fondées sur les effets fictionnels, sur le « comme si » de la mise en discours et du travail d'écriture poiétique à partir du référent spatiotemporel offert par un environnement naturel et social inscrit dans le flux du temps physique et cosmique.

Paul Ricœur semble donc être victime non seulement de sa dépendance à l'égard du paradigme narratologique des années 1970, mais aussi de sa fascination pour la phénoménologie métaphysique de Martin Heidegger. En situant la mise en intrigue du discours historique en tant qu'opération de "tiers-temps" non pas dans une séquence de mouvements de configuration à partir de notre manière de vivre l'espace-temps physique, mais dans la refiguration discursive tout en gommant les repérages spatiaux de toute configuration historique, le philosophe herméneute entretient un malentendu fondamental quant à son effort de résoudre l'immanquable aporie de la phénoménologie du temps par la définition d'une situation médiatrice entre histoire et fiction. Cette équivoque est issue notamment du silence quant aux effets fictionnels de toute usage de la langue et de toute mise en discours alors que ceux-ci contribuent, eux aussi, à relativiser fortement le statut ontologique accordé à la temporalité du Dasein. Ce premier grand malentendu a pour corollaire pas moins de quatre autres malentendus.

\section{La double articulation du temps calendaire}

Découlant de la position singulière assignée par Paul Ricœur au «tierstemps ", le deuxième malentendu à signaler est entretenu par la notion du «temps calendaire» telle qu'elle est empruntée à Émile Benveniste dans la

9. Ricœur 2000 : 302-307 et 359-369. La référence dans la longue note 77 (367-369) à la notion de "Vertretung" développée par Hans-Georg Gadamer dans Wahrheit und Methode est significative d'une herméneutique philosophique qui, ignorant le linguistic turn, est incapable de saisir les effets de la "poiétique" propre à la mise en discours verbale; cf. Calame 2002 : 67-77.

10. Cf. en particulier Chartier 1998 : 91-99. 
quête du pont à lancer entre temps vécu et temps cosmique. Selon les propositions formulées naguère par l'éminent linguiste comparatiste, le temps du calendrier serait en effet caractérisé par l'organisation linéaire dessinée par un comput partant d'un point axial d'origine. À partir d'un point correspondant en général à un événement fondateur, une scansion homogène et dynamique orienterait donc un temps "chronique"; ce temps est pris par Paul Ricœur pour l'exemple même d'un «tiers-temps " permettant au temps psychique de s'articuler sur le temps cosmique ${ }^{11}$.

Or il s'avère que dans toute communauté culturelle, la temporalité du calendrier est en fait fondée sur une combinaison de conventions qui portent non seulement sur une mesure régulière permettant de se déplacer de manière linéaire dans le passé, mais aussi sur une série de récurrences d'ordre plus ou moins cyclique. Certes, en vertu du repérage à l'égard d'un moment fondateur institué en point axial, le temps calendaire dans sa dimension linéaire dépend de la représentation que chaque société se fait de son passé communautaire ; dans ses effets pratiques, la temporalité du calendrier correspond dans cette mesure à une manière de vivre collectivement, intellectuellement et symboliquement la progression et la fuite inexorables du temps cosmique. En revanche, dans sa dimension cyclique, le temps calendaire dépend notamment des repères que fournit le monde «naturel » avec des alternances d'ordre astronomique ou des répétitions météorologiques tels que le cycle des saisons ou l'alternance des moussons, mais aussi avec les successions biologiques récurrentes attachées à la mortalité de l'homme. Par le rythme qu'il impose au comportement social, le temps du calendrier fonde également une manière communautaire de vivre, par la pratique symbolique et culturelle, l'aspect cyclique du temps organique. C'est donc en raison de sa double dimension, à la fois linéaire et circulaire, que le temps calendaire fournit effectivement, avant l'histoire et ses effets fictionnels, une médiation privilégiée entre d'une part l'espace-temps physique, biologique et cosmique, et d'autre part le temps psychique et social. Avant l'intervention de l'écriture de l'histoire, l'une des médiations temporelles recherchées s'opère dans cette scansion symbolique complexe à partir d'un environnement spatio-temporel spécifique et de représentations collectives du passé dans son espace de déploiement.

Tout en organisant un espace social, le temps calendaire offre ainsi une temporalité composite. Sans doute est-ce dans sa réalisation que s'opère la conjonction entre toutes les représentations culturelles du passé que nous classons dans la catégorie floue de "mythe» et les pratiques symboliques étude intitulée "Le langage et l'expérience humaine » et publiée à l'origine dans Diogène 51, 1965 : 3-13). 
réglées et récurrentes que nous plaçons sous l'intitulé, non moins vague, de « rite». Le premier ne s'oppose pas au second, comme le pense notamment Paul Ricœur. Mais la représentation narrative du passé avec ses repérages spatiaux, socialement partagée, se combine avec la pratique somatique communautaire, inscrite dans la récurrence calendaire : ces gestes rituels sont attachés à des lieux précis pour donner une profondeur symbolique et pratique supplémentaire à la scansion d'un temps qui n'a rien d'ordinaire et qui de mépris ne peut susciter que celui d'un philosophe métaphysicien ${ }^{12}$. Inscrits dans le temps calendaire, « mythe » et « rite » contribuent à transformer spatialement et socialement, par différents moyens sémiotiques, les composantes aussi bien linéaire que cyclique du temps physique et biologique, dans son écoulement et dans ses récurrences. Ce faisant, les différentes manifestations symboliques que l'anthropologie moderne place sous les étiquettes de mythe et de rite transforment en un savoir partagé les appréhensions individuelles préfigurées du temps et de l'espace du monde; des préfigurations qui sont ressenties comme temps et espaces psychiques à partir du développement du temps physique et du déploiement de l'espace organique. On est bien loin du temps «vulgaire » rejeté par Heidegger au profit de l'« historialité » qui serait constitutive de la projection du Dasein dans la mort.

\section{La question de la mise en discours}

Dans cette perspective, force est de constater par ailleurs qu’à partir des préfigurations de la temporalité que représentent nos sentiments individuels, biologiques et psychiques, mais aussi socialisés du temps physique et cosmique, la configuration d'un temps social par l'intermédiaire d'un temps calendaire implique d'emblée une mise en discours. On pourra donc s'imaginer que l'histoire en tant que savoir partagé se fonde notamment sur nos perceptions et nos manières de vivre pratiquement l'histoire en tant que passé, mais que ce savoir est aussi constamment remodelé par les historiens à l'aide de moyens d'ordre essentiellement discursif. Si les structures narratives semblent bien transformer par leurs logiques toute histoire en récit, le moment de configuration temporelle et spatiale que représente l'élaboration de l'historien doit être étendu à toutes les procédures de la mise en discours déjà évoquées, des modes de la schématisation aux tournures rhétoriques en passant par les différentes formes de l'argumentation ${ }^{13}$.

12. Ricœur 1985 : 154-156. Les concepts relatifs et opératoires, mais souvent naturalisés et transformés en universaux que sont devenus le mythe et le rite ont fait l'objet de plusieurs critiques que j'ai reprises en 1996a: 12-25, et 1996b : 15-29.

13. Le rôle des figures de rhétorique dans la mise en discours historiographique a finalement été reconnu par Ricœur (2000 : 320-338) (cf. supra n. 8). Il en dit non seulement les fonctions argumentatives, mais aussi le pouvoir figuratif, producteur d'images (2000: 349-358). 
En intégrant au moment de la configuration historique les différentes procédures fondant la mise en discours en général, on retrouve la conception classique hellène de la poétique comme fabrication, de la poétique comme "poiétique" : poieîn fondé sur les opérations de la mímesis fictionnelle telle que l'envisage Aristote et telle que la reprend Paul Ricœur dans le premier volet de sa première enquête sur le temps pour la focaliser sur la mise en intrigue de l'action et pour la restreindre ainsi essentiellement aux procédures de mise en forme narrative ${ }^{14}$. Dans cette perspective po(i)étique plus large sur laquelle on aura l'occasion de revenir, le travail de l'historien peut être conçu comme une "historiopoiésis " effaçant la distinction que l'on pourrait être tenté de tracer entre histoire et historiographie, entre histoire comme faire et histoire érudite (configuration d'un côté, refiguration de l'autre ?), donc comme la construction et le façonnement de temporalités préfigurées dans nos appréhensions individuelles et collectives du temps et assorties de repérages spatiaux. Devenu « historiopoiète", l'historiographe peut naturellement être appelé à relire un passé à la configuration discursive duquel lui-même ou d'autres ont activement participé en phase de mimèsis II ; il intervient donc aussi, en "herméneute", dans les opérations de refiguration de mimèsis III. Mais si herméneutique il y a, elle est dynamique, destinée non pas à retracer le sens d'un texte fixé comme le sont l'Ancien ou le Nouveau Testaments, mais à produire un discours nouveau, qui n'est pas de l'ordre du simple commentaire.

Dans leur dimension discursive, configurations et refigurations temporelles et spatiales se fondent ainsi sur les procédures de sélection, de schématisation, de mise en séquence argumentative, de concaténation logique, de description et de modélisation par prototypes ou stéréotypes interposés qu'on a dites caractéristiques, en plus des structures narratives, de toute mise en discours. C'est dans cette mesure que, dans la phase ultime de la réflexion de Paul Ricœur sur le temps métamorphosé en histoire, les schématisations que constituent les mentalités, les variations d'échelle ou les représentations elles-mêmes peuvent être incluses dans la phase intermédiaire d'explication-compréhension. Au début des années 1970 déjà, Reinhart Koselleck avait parfaitement saisi que, davantage qu'aux simples formes narratives, il appartient aux concepts partagés d'assurer l'intelligibilité de l'histoire et donc du temps passé :

«Les concepts qui englobent des faits, des rapports complexes et des processus passés deviennent, pour l'historien qui se sert d'eux dans sa démarche cognitive, des catégories

14. J'ai tenté d'expliciter ce trait fondamental de la po(i)étique grecque, dont on peut tenter de rendre la fonction de fabrication par l'emploi du terme fictionnel, en 2000b : 38-51; le travail dans ce sens de quelques «historiopoiètes » hellènes est esquissé dans l'ouvrage de 1996b : 30-46; cf. aussi 2000b : 145-161, et infra n. 17. 
formelles qui peuvent être posées comme conditions de possibilité d'histoires. Ce n'est qu'avec des concepts susceptibles de couvrir une certaine durée [...] que s'ouvre la voie qui permet de savoir comment une histoire jadis "réelle" peut aujourd'hui nous apparaître comme possible et ainsi représentable $»^{15}$.

C'est en tant que poíeseis discursives, configurantes et refigurantes, que les pratiques des historiens contribuent à la stabilisation des temporalités collectives plurielles que connaît toute communauté politique et culturelle, dans un "comme si » qui assure à la fois la relation de référence aux événements localisés dans le passé et leur compréhension dans le présent.

\section{La dimension énonciative}

Puisque la constitution du temps (et de l'espace) en histoire au sens large du terme relève en particulier des différentes procédures de la mise en discours, une quatrième équivoque peut naître de l'absence de prise en compte de la dimension énonciative propre à tout acte de discours; et cela de nouveau à partir d'une définition trop restrictive du temps calendaire. À propos de la sélection, de la succession et de l'enchaînement logique et argumenté que nous pensons devoir être constitutifs de la temporalité de l'histoire, Émile Benveniste (1974: 71) affirme que :

"Dans toutes les formes de culture humaine et à toute époque, nous constatons d'une manière ou d'une autre un effort pour objectiver le temps chronique. C'est une condition nécessaire de la vie des sociétés, et de la vie des individus en société. Ce temps socialisé est celui du calendrier ».

Institué comme on l'a vu en temps calendaire, ce temps de notre expérience partagée et culturelle de l'histoire serait donc scandé par un comput régulier à partir d'un point d'origine coïncidant avec un moment fondateur du passé de la communauté. Or, dans la perspective adoptée par Émile Benveniste, ce temps compté non seulement n'intègre pas dans sa linéarité des récurrences cycliques dont est aussi fait le temps du calendrier, mais il s'opposerait au "temps linguistique", lié à l'exercice de la parole. Il y aurait opposition entre temps calendaire et temps linguistique dans la mesure où, à l'évidence, le point axial de ce dernier, issu de la mise en discours, coïncide non pas avec un point d'origine, mais avec le moment même de son énonciation : un "maintenant " qui se définit en concomitance avec un "ici", dans une nouvelle imbrication des paramètres temporels et spatiaux ; un marquage d'ordre temporel qui se combine avec un repérage spatial et avec l'intervention du je du locuteur pour

15. Ricœur $2000: 231-238$ et 241-277 ; Koselleck $1990: 141$; sur les procédures schématisantes et rhétoriques du «comme si » de tout discours en sciences humaines, voir les références que j'ai données en 2002: 67-77. 
constituer ce que Émile Benveniste appelle dans une autre étude l'«appareil formel de l'énonciation ${ }^{16}$.

Paradoxe surprenant que cette opposition entre temps calendaire et temps linguistique pour qui se sent sollicité par les représentations du temps et de l'espace. Il semble en effet que la saisie configurative du temps et de l'espace implique au contraire, dans les opérations complexes de la mise en discours, une combinaison entre temps calendaire ou chronique et temps linguistique. En configurant par des moyens linguistiques un temps à la fois lissé, rythmé et profilé par un système de mensuration homogène, toute représentation discursive du temps oriente la ligne temporelle organisée à partir du point d'origine du comput vers le point focal de la mise en discours. C'est dire que, par la mise en discours, la temporalité organisée par le comput depuis un point axial d'origine également repéré du point de vue spatial est orientée vers l'" instance d'énonciation " qui est elle-même saisie dans ses paramètres spatiaux et temporels; ceux-ci correspondent au hic et nunc de l'énonciation, puis aux différents moments spatio-temporels de la réception. Répondant à une chronologie particulière, la mise en discours du temps (et de l'espace) que constitue l'écriture de l'histoire est donc également orientée par une logique énonciative.

Relative à l'opération de mise en discours du temps avec son corollaire spatial, la tension existant entre les deux points focaux de toute temporalité discursive, entre point d'origine chronologique et instance d'énonciation dans le hic et nunc de la communication, implique un double passage : de l'ordre de la réalité sociale à celui du discours, puis, en retour, du discours à cette réalité; de l'extra- à l'intradiscursif, puis de l'intra- à l'extradiscursif. Du point de vue temporel, on passe ainsi de l'échelle de mesure du temps organisé et configuré socialement à l'assomption discursive et énonciative de cette scansion pour revenir à la réalité et aux représentations sociales par l'intermédiaire du processus de la communication, dans son hic et nunc mobile. Ce mouvement discursif en tension exige que l'on distingue soigneusement, du point de vue temporel aussi bien que du point de vue spatial, deux niveaux d'ordre sémiotique différent: d'une part (sur le plan extradiscursif), celui qui correspond à l'acte de l'énonciation et de la narration, c'est-à-dire au temps et à l'espace empiriques et historiques de la mise en discours qui sont aussi ceux du raconter; d'autre part (dans l'ordre de l'intradiscursif), celui de l'expression dans le discours lui-même, par différents moyens linguistiques et énonciatifs, de ce temps et de cet espace de l'énonciation (et par conséquent de la narration). Cette

16. Benveniste 1974 : 76-78, ainsi que 79-88 sur l' "appareil formel de l'énonciation "; voir aussi, en rapport avec la temporalité, Coquet $1997: 81-103$. Pour des références à différents types de comput à partir d'un moment d'origine, voir Molet 1990 : 257-261. 
manifestation d'ordre discursif du temps et de l'espace correspond au moment et au lieu de l'énonciation énoncée (ou de l'énoncé de l'énonciation ); c'est une réalité intradiscursive à distinguer de la réalité spatiotemporelle et sociale d'ordre extradiscursif, même si la première se trouve dans une forte dépendance par rapport à la seconde. Pour l'historien, cela signifie que le moment et le lieu de sa mise en discours et de son énonciation sont inclus dans l'opération de configuration du temps et de l'espace de l'histoire. En raison même de l'évidente perméabilité entre extra- et intradiscursif, notamment par le processus de la communication, le discours historique lui-même porte les traces (énonciatives) de cette tension énonciative constitutive de toute mise en discours et donc toute référence par les moyens de la langue.

C'est ainsi dans l'instant même de la mise en discours, avec les opérations propres à la mimèsis II, que le temps et l'espace du vécu dans leurs dimensions sociales et individuelles interviennent dans la configuration de tout discours, notamment s'il est poétique et littéraire; et cela aussi bien dans le rythme de l'énonciation (et plus particulièrement dans celui de la narration) que dans la scansion chronologique de l'énoncé lui-même (par conséquent dans celle du récit). Ainsi, à la transition qu'opère la mise en discours entre la situation empirique et extradiscursive de l'énonciation (avec sa référence au temps et à l'espace sociaux) et la configuration énoncée et désormais intradiscursive de cette situation, la bonne méthode sémio-narrative exige d'ajouter un partage tout aussi instrumental entre le temps de l'énonciation de l'énoncé, avec son rythme et sa logique propres, et le temps de l'énoncé lui même ; ce temps est en général le résultat d'une reconstruction dans le sens d'un déroulement linéaire. C'est ainsi que, quand l'énoncé prend une tournure narrative, on peut restituer en tant que distinction subordonnée le partage également opératoire entre Erzählzeit et erzählte Zeit (Günther Müller), entre temps de la narration et temps raconté ${ }^{17}$.

Aussi artificielle et opératoire qu'elle puisse être elle-même, la séparation déterminante entre ce qui est extérieur au texte issu de la mise en discours et ce qui est de l'ordre intradiscursif dans la fabrication par et dans le discours contribue donc à subordonner et à fortement nuancer les distinctions désormais traditionnelles entre Erzählzeit et erzählte Zeit, entre «temps de l'énonciation [narrative] " et «temps de l'énoncé [narratif]» (Gérard Genette), voire entre « discours » et « histoire» (entendue comme

17. Cette distinction a été retravaillée par Ricœur (1984: 113-131). Dans mon étude de 2000c : 399-412, consacrée à l'analyse spatio-temporelle d'un poème rituel de Bacchylide, on trouvera des précisions sur les niveaux temporels d'ordre discursif indiqués ici; voir également à ce propos Borutti (1996: 250-252). 
« récit » selon Émile Benveniste) ${ }^{18}$. En effet, focalisées sur la narration, ces distinctions opèrent un partage d'ordre uniquement intradiscursif entre la chronologie du récit et sa conduite narrative, entre sa logique organisée selon la ligne de l'écoulement du temps et le rythme qui, propre à la narration, est fait d'extensions, de condensations, d'analepses, de prolepses, etc. Tout en tentant de faire la synthèse de distinctions qui ne sont pas entièrement homologues et en les reprenant ainsi à son compte, Paul Ricœur a fait un premier pas pour rompre le principe d'immanence sousjacent à cette perspective narratologique d'inspiration structurale. Dans cette perspective, l'expérience narrative du temps née du monde du texte se réfere à la Zeiterlebnis. Proposé par Günther Müller lui-même, ce retour au temps du vécu devrait rendre compte par exemple du sens même de $\grave{A}$ la recherche $d u$ temps perdu; dans ce cas, le travail poiétique et pratique de l'écrivain commence au moment même où se clôt l'expérience fictive du temps (Ricœur 1984: 119-131, 150-151, 217-234). Sans doute est-ce en raison de ce mouvement contradictoire de constante anticipation rétrospective sur le moment de la mise en discours que l'œuvre de littérature, à l'exemple de l'orientation temporelle de La Recherche, semble "s'ouvrir" sur un extérieur seulement au moment de sa reprise et de son appropriation en lecture. L'ouverture extradiscursive ne s'effectuerait qu'au moment de la refiguration, dans le mouvement de la mimèsis III.

De là la tension indiquée entre le point axial du hic et nunc de l'énonciation et le point d'origine du temps mesuré et raconté. Il est donc opportun de confirmer le caractère fondamental de la distinction opératoire indiquée entre le temps et l'espace empiriques de l'acte d'énonciation et les temporalités ainsi que les spatialités discursives de l'énonciation énoncée, puis du récit : avec sa double scansion, linéaire et cyclique, le temps social du calendrier produit dans le discours même les repères spatiaux et temporels dans la tension indiquée entre le point d'origine du temps et de l'espace racontés et le hic et nunc d'une communication souvent ritualisée.

\section{L'incontournable pragmatique}

Toute configuration du temps passé par une mise en discours ne peut que reformuler une temporalité déjà préfigurée et en partie configurée dans d'autres formes d'expression pour revenir, comme dans tout processus d'ordre symbolique, sur la réalité déjà informée dont elle tire son

18. Voir successivement Müller 1968: 269-286, Genette 1973: 71-78 et 128-130, Benveniste 1966: 237-250, ainsi que 251-257, et 1974: 79-88, pour la relation entre le "discours " et l'«appareil formel de l'énonciation", avec les remarques critiques d'Adam, Revaz \& Lugrin 1998. Sur les effets constituants du "temps discursif » dans l'écriture de l'histoire, voir aussi de Certeau 1975 : 104-109. 
origine $^{19}$. C'est dans ce retour que se niche une nouvelle équivoque entretenue par la volonté de situer l'intervention de l'historien et la « représentance " du côté de la refiguration et de les comprendre par conséquent comme mimèsis III. À faire coïncider l'écriture de l'histoire avec ce moment interprétatif, et en général critique, on risque de manquer sa dimension pragmatique, c'est-à-dire de figer les discours historiographiques du passé en des textes immuables, uniquement susceptibles d'une herméneutique de type biblique. Or les procédures de mise en discours qui résultent des opérations configuratives de l'historiographie se trouvent en constante interaction avec le flux de l'histoire elle-même. Inscrites dans le mouvement historique dont elles proviennent, ces mises en discours ont donc en retour un impact immanquable sur l'orientation de ce mouvement. Si l'on tient à placer ce retour pragmatique des procédures de la configuration historienne du côté de mimèsis III, on dira que la refiguration ne consiste pas uniquement en lectures interprétatives des manifestations de l'historiopoiésis, mais aussi en pratiques sociales. Le travail de configuration et de reconfiguration temporelles (et spatiales) sur le passé suscite donc, en combinaison avec des réélaborations d'ordre intellectuel, une herméneutique pratique.

Ainsi, dans l'Antiquité comme dans la modernité, les lieux de mémoire sont l'objet de célébrations destinées à réactiver régulièrement, dans le présent et dans une herméneutique communautaire, l'histoire qu'ils signalent et qu'ils désignent ${ }^{20}$. Ils constituent à cet égard les points de repère spatiaux du "tiers-temps " qu'est le temps calendaire dans sa double dimension, linéaire et cyclique, entre temporalités sociales et temporalités discursives. Mais ces sites évocateurs d'un passé sont eux-mêmes soumis à une historicité qui les transforme et les remplace à mesure que se modifie la relation mémoriale du groupe concerné avec son propre passé. Dans leur organisation géographique, les lieux de mémoire apportent une contribution spatiale et visuelle déterminante à la fixation temporaire des représentations et configurations du passé dont l'histoire est faite; ils constituent aussi les traces matérielles de la relation pratique du travail des historiens avec la conjoncture présente. L'intérêt pour l'histoire, la sensibilité individuelle et communautaire pour le passé se nourrissent sans conteste des pratiques érudites et didactiques des historiopoiètes. Les

19. C'est là le propre de toute manifestation dépendant du processus de la construction et de la représentation symboliques; voir à ce sujet les propositions que j'ai pu avancer en 1996b : 15-68 (en particulier 49-54).

20. La question du rôle historiographique assumé par les sites de la mémoire communautaire fait l'objet de la mise au point introductive à l'entreprise collective animée par Nora 1984 : XIXXXXII. 
célébrations rituelles régulières autour des lieux de mémoire que sont en Grèce classique les tombeaux héroïques, de même d'ailleurs que les commentaires ponctuant les témoignages exposés dans les musées-monuments de la modernité, sont là pour assurer la relation herméneutique et pratique entre les représentations plurielles du passé construites par les historiens et une mémoire collective à entretenir et à adapter aux circonstances d'un présent toujours en mouvement! On ne saurait donc être historien d'une communauté différente, sans en être également l'anthropologue.

\section{Pour une poétique des indices historiographiques}

L'aspect à la fois spatial, référentiel et pragmatique des temporalités discursives nous a donc très sommairement conduits aux lieux de mémoire, c'est-à-dire en aval de la mise en discours de l'historiopoiésis. Si l'on se reporte en amont, on se trouve évidemment confronté à des espaces naturels et construits qui, préfigurés et retenus dans les mémoires, individuelles et collectives, sont configurés dans le discours de type historique pour être entraînés et transformés dans la configuration temporelle de l'opération historiopoiétique. Du point de vue spatial, le travail de l'historiopoiète se fonde notamment sur des indices matériels qu'il est convenu de saisir en termes de traces; par leur nature concrète et sensible, les traces correspondent en particulier à des espaces signifiants. Mais qui dit trace, dit aussi témoignage et document. La réflexion des premiers historiographes grecs, en particulier celle de Thucydide, sur ces signes matériels (semeîa) qui sont aussi des indices de reconnaissance servant de preuves (tekméria) et d'attestations (martúria), se combinant en cela avec les discours de la tradition orale, est si riche que j'entends y revenir dans une autre étude. Quel que soit leur statut sémiotique, les documents traités en indices, en preuves et en garants relèvent, dans la perspective de l'historien et de son travail de configuration historiographique, des préfigurations de mimèsis I. Cela ne les empêche pas de présenter des traits de configuration spatiotemporelle qu'une critique de l'historiopoiésis ne saurait négliger.

\section{Vue et ouïe : histoire récente}

C'est en particulier sur ce point d'une mise en forme préalable des indices du passé, à titre de préfiguration, que la première historiographie grecque peut être éclairante. L'aspect indiciel du passé se manifeste notamment dans la distinction que dessinent les historiens hellènes classiques entre les actes anciens (tà palaiá) et les actes "nouveaux" (tà kainá). Reportons-nous, avec Hérodote, au moment où les Athéniens revendiquent face aux Tégéates la place d'honneur dans l'ordre de la future 
bataille de Platées. Aux guerres anciennes menées contre les Héraclides, contre les Amazones, ou à l'occasion de l'expédition en Troade, les Athéniens ajoutent le haut fait que représente la toute récente bataille de Marathon. Le partage entre passé éloigné et passé proche dans la définition du champ de l'histoire se double d'une distinction entre ses acteurs : héros individuels d'une part, tels Minos, Agamemnon ou Thésée dans l'«archéologie» de Thucydide, communautés civiques des Athéniens, des Péloponnésiens ou des Barbares de l'autre. Mais quand, plus d'un siècle après la fin des guerres médiques, Démosthène reprend la même distinction entre événements anciens (arkhaîa kaì palaiá) et événements récents (kainá), la limite temporelle départageant les seconds des premiers s'est naturellement déplacée. Ainsi, dans l'argumentation sur la nécessité pour Athènes d'entretenir une flotte, la bataille de Salamine, qui appartient elle aussi au temps des guerres médiques, est passée du côté des palaiá alors que la toute récente capitulation des Thébains, grâce à l'intervention des trières athéniennes en Eubée, appartient de toute évidence aux kainá. Or il faut ajouter que pour Démosthène, la distinction entre passé éloigné, si glorieux soit-il, et passé récent recoupe la ligne de démarcation tracée par Hérodote lui-même, quant aux modes de son enquête, entre ouïe et vue. Si le savoir sur le passé de la cité "dont le temps lui-même ne saurait effacer la mémoire (mnéme)» se fonde pour le public de l'orateur sur l'ouïe (akoé), sa connaissance de l'événement récent se fonde sur "ce que tous vous avez vu (heorakáte) " ${ }^{21}$.

Ce que nous souffle cette conception antique du temps historique, c'est donc un partage perméable entre un passé assez éloigné pour qu'il ne nous soit connu que par des lógoi, par des dires transmis par la tradition (en général orale), et un passé récent dont l'historiographe et son public ont été les témoins oculaires. Formulations verbales d'un côté, évocations d'images de l'autre. Lorsque la tradition écrite deviendra la garante de la mémoire de la cité, la préfiguration du passé éloigné dépendra de la mise en discours qu'implique le document verbal écrit alors que la préfiguration de l'histoire plus proche continuera à reposer sur des témoins oculaires ; ces derniers se chargeront de traduire sous forme verbale et orale les images alimentant leurs souvenirs. À l'égard de ce partage entre histoire ancienne et histoire récente, la multiplication des médias a rendu la situation de l'historiographie moderne particulièrement délicate. Ainsi en va-t-il en

21. Hérodote, Histoires, 9, 27, 1-6 et Démosthène, Contre Androtion 12-15: cf. Calame 1996a: 39-43. À la suite du passage de l'«archéologie " que Thucydide consacre au synécisme d'Athènes $(1,16,1)$, l'historiographe est conduit à diviser lui aussi en trois périodes l'espace temporel s'étendant du moment de la fondation d'Athènes en tant que cité jusqu'à la guerre présente : tà arkhaîa, tà hústeron, mékhri toûde (!) tô̂ polémou. 
particulier des termes du débat sur les camps d'extermination et sur ses sinistres prémisses politiques. Pour l'instant, ce point de non-retour de l'histoire européenne appartient à ce que les historiens grecs classiques appelleraient un kainón. Le rôle qu'y jouent les témoins oculaires et, par images interposées, les témoignages visuels est encore déterminant. L'intervention nouvelle de la photographie, de l'archive filmée et de l'enregistrement du témoignage oral laisse supposer qu'il en sera ainsi au-delà de la disparition des derniers rescapés et rescapées d'un plan d'anéantissement qui fut conçu et appliqué de manière d'autant plus cynique et systématique que sa réalisation a précisément bénéficié des moyens techniques de l'ère industrielle. Certains d'entre eux ont d'ailleurs permis, de manière subséquente, d'en maintenir la mémoire visuelle et auriculaire.

Quel que soit le statut sémiotique, sinon cognitif ou neuronal, d'images souvent traduites en termes verbaux, le moment stratégique est en fait celui du passage de ces différents modes de la préfiguration des épisodes les plus marquants, et souvent les plus douloureux du temps passé, à sa configuration pragmatique par les procédures mises en ouvre par les historiographes et historiopoiètes. Pour penser cette transition, on peut à nouveau se laisser inspirer par les termes et concepts hellènes. Thucydide - on l'a signalé rapidement - fonde sa recherche d'indices (semeîa) destinés à devenir des preuves (tekméria) sur des témoins et témoignages: des mártures et des martúria entendus comme garants. Mais Hérodote conçoit son propre travail de réélaboration narrative et de réécriture descriptive à partir des discours (lógoi) des autres comme un travail d'enquête (historía). Souvent interrogé, le sens du terme hístor renvoie sans doute plus à l'idée de la garantie offerte par celui qui recueille les témoignages qu'à celle d'attestation oculaire que semble impliquer la racine du terme; on sait en effet que le fondement étymologique d'histoire, par l'intermédiaire de la racine vid-, évoque aussi bien le videre latin que le voir français. Si donc le mártustémoin est bien le garant de ce qu'il a vu, l'histor-savant est susceptible d'arbitrer entre plusieurs témoignages dont il se porte garant. Tel est d'ailleurs le statut énonciatif du discours d'Hérodote : rapportant souvent plusieurs versions narratives (lógoi), l'historien d'Halicarnasse ne prend que rarement position à leur égard ${ }^{22}$. Hérodote est enquêteur et garant avant d'être juge. C'est pourquoi, tout en assignant à son enquête la visée mémoriale qui est celle de la poésie épique, il renonce à présenter un discours de vérité inspiré

22. Le sens de historía et les postures énonciatives qu'entraîne l'attestation qui en découle sont évoqués par Marincola 1997 : 3-10, Calame 2000b : 115-125, et Hartog 2001 : 24-35 et 407-411 (voir aussi 2000 : 6-7, pour la différence sémantique entre mártus et histor). Les aspects judiciaires du discours historiographique grec ont été éclairés par Darbo-Peschanski 1998 (172-175 pour Hérodote) alors que Prost 1996 : 288-293 a étendu la réflexion à l'écriture de l'histoire en général. 
par la Muse et, dans la plupart des cas, il préfere dissimuler son autorité énonciative derrière le on en général pluriel ou anonyme des récits des autres; il laisse ainsi souvent à ses auditeurs le soin du jugement (moral) de véridiction dans le moment herméneutique de la refiguration.

\section{Retour à la "poiétique"}

Entre la mise en discours par le témoin de ses souvenirs visuels et leur saisie ainsi que leur reformulation discursive par le garant et arbitre, on est donc passé de la préfiguration à la configuration - de mimèsis I à mimèsis II, pour reprendre encore une fois les concepts empruntés à Aristote et développés par Paul Ricœur. Or c'est précisément le philosophe grec qui montre, dans la Poétique, quels sont les aspects de fabrication propres à toute représentation narrative, qu'elle soit dramatisée ou non. Le poète est donc conçu comme un artisan d' «intrigues" (mûthoi). N'y a-t-il pas dès lors contradiction avec la célèbre distinction, tracée dans le même chapitre de la Poétique et si fréquemment alléguée ? Au début d'un développement consacré à l'art de la po(i)étique, Aristote ne distingue-t-il pas précisément le poieîn du légein, la "création " de la simple " relation " ? En contraste avec l'aspect mimétique et représentationnel de l'art du poète, l'auteur de la Poétique n'attribue-t-il pas à l'enquêteur (ho historikós) la fonction de simplement «dire» ce qui s'est passé (tà genómena)? Indépendamment de la forme rythmée ou non de la diction, il reviendrait ainsi à l'historien de dire ce qui est advenu, mais au poète de dire les choses "telles qu'elles pourraient advenir ". Au premier serait réservé le particulier (kath’hekástou), au second le général (kathólou). Au premier, les actions des hommes qui, identifiés par un nom propre, ont réellement agi ou pâti, tel Alcibiade; au second les actions humaines qui, parfois attribuées à des individus, s'inscrivent dans l'ordre de la vraisemblance ou de la nécessité ${ }^{23}$.

Néanmoins, en comprenant toute opération de mimèsis comme l'effet du poieîn, Aristote nous invite à élargir à d'autres formes de mise en discours les modes de la représentation attachés à la mise en intrigue narrative et poétique. En tant que tel, l'art poétique porte - on le sait - sur les possibles (tà dunatá) et par conséquent sur ce qui pourrait advenir selon l'ordre du vraisemblable ou du nécessaire. Mais Aristote ne manque pas d'ajouter que l'artisan d'intrigues (tôn múthon poietés) qu' est le praticien de l'art poétique peut aussi façonner des actions passées (genómena):

23. Aristote, Poétique 9, 1451a 36-b 11, relu notamment par Ricœur 1983: 57-84; voir les remarques et les références que j'ai tenté d'offrir dans les études citées supra n. 10 : pour l'histoire, on y ajoutera les observations de Borutti $1996: 240-248$, et pour l'anthropologie les observations de Borutti 1999 : 99-116. 
"Il apparaît donc que le poète (poietés) doit être créateur d'intrigues davantage que de rythmes. S'il est en effet poète, c'est par la représentation (mimesis) et ce qu'il représente ce sont des actions (praxeîs). S'il lui arrive de fabriquer (poieîn) des actions advenues (genómena), il n'en est pas moins poète. Car rien n'empêche que certains parmi les événements passés soient tels qu'ils s'inscrivent dans l'ordre de la vraisemblance (eikós) et du possible (dunatá), et par intermédiaire cet homme en est le poète ${ }^{24}$.

Sans prétendre ajouter un commentaire propre à un chapitre si souvent commenté, force est de reconnaître que la remarque conclusive d'Aristote est rarement mentionnée. Il n'y a rien de très étonnant à cette omission puisqu'une telle affirmation tend à effacer la distinction largement ressassée entre enquête historique et création poétique, entre relation du singulier et représentation du général. Que l'on partage ou non l'avis d'Aristote sur la relation entre l'advenu d'une part, et le possible, le vraisemblable ou le nécessaire de l'autre, le passé peut faire lui aussi l'objet d'une élaboration "poiétique", au sens étymologique du terme.

Hérodote et Thucydide sont là pour nous montrer que les logographes sont aussi des historiopoiètes. La création "poiétique" d'un monde possible à partir d'une réalité préfigurée dans l'enchaînement nécessaire conduisant à la construction d'une vraisemblance correspond en somme aux processus sous-jacents au concept moderne du «fictionnel»; non pas au sens de la création d'un monde de fiction pourvu d'une existence sémantique autonome (en correspondance avec le sens de fictional en américain), mais au sens de la fabrication d'un monde possible à la fois à partir de l'expérience sensible et partagée du monde naturel et social, et par l'exploitation des potentialités sémantiques de toute langue (pour revenir sur ce monde de la pratique sociale). Rappelons qu'à propos des discours dont il ponctue son récit, Thucydide lui-même précise qu'ils sont restitués selon les paroles probablement exigées par la situation du moment et dans le respect, quant à l'opinion exprimée, des mots vraiment prononcés ${ }^{25}$.

Sans doute l'histoire ne porte-t-elle ni sur l'universel, ni sur le singulier, mais sur le spécifique qui seul peut être compris dans la mesure où il renvoie à une intrigue. Du moins est-ce là la définition proposée par Paul Veyne. S'il est également vrai que la mise en intrigue permet d'établir les relations nécessaires à la compréhension et que par conséquent «l'enchâ̂nement du texte exprime les imbrications réelles des causes, des conditions, des raisons et des régularités ", alors l'historiopoiésis est aussi justiciable du travail de représentation et de fabrication indiqué par la notion aristotéli-

24. Aristote, Poétique 9, 1451b 27-32 (traduction de Roselyne Dupont-Roc \& Jean Lallot, adaptée), où l'expression kaì dunatà genésthai est parfois athétisée par les éditeurs modernes.

25. Thucydide 1, 22, 1. La différence entre un "fictional world» et un monde possible créé par des moyens poétiques est bien redéfinie par Edmunds 2001 : 95-107; voir encore supra n. 8. 
cienne de la mímesis poétique ${ }^{26}$; à cette différence près néanmoins que l'historiographe représente les actions des hommes et des groupes sociaux non pas comme elles pourraient ou devraient advenir, mais comme il pense les rendre semblables au vrai et intelligibles après qu'elles sont advenues. L'historien moderne fait intervenir mobiles, intentions, buts et circonstances dans des actions sélectionnées et schématisées pour être mises en séquence et inscrites dans des espaces à leur tour schématisés. Ainsi l'historiographe abandonne-t-il le singulier pour se faire le poietés du passé. Tel est l'un des sens de l'œuvre de configuration historiographique à partir des préfigurations du temps et de l'espace et de leur matérialisation dans différents indices. L'impact pragmatique de l'écriture historienne du temps et de l'espace sociaux est sans doute à ce prix poiétique.

\section{Pour une historiographie anthropologique}

S'agissant du développement de la puissance d'Agamemnon, et sa capacité d'organiser une expédition navale préfigurant la puissance maritime d'Athènes, Thucydide n'hésite pas à se fonder sur le témoignage d'un poète tel que Homère. L'aède de la guerre de Troie doit en effet être considéré comme susceptible de fournir des indices, et par conséquent des preuves (tekmeriôsai). Mais pour remonter plus haut dans le temps et pour comprendre les causes de la puissance d'Agamemnon, il convient de suivre un parcours généalogique. Par l'intermédiaire d'Atrée, le roi de Mycènes, on parvient ainsi jusqu'à Pélops, le héros fondateur et éponyme du Péloponnèse. Dans cette quête d'un point axial du point de vue à la fois temporel et spatial, l'historiographe n'a plus d'autre choix que celui de laisser les poèmes homériques pour se tourner vers ce que nous appellerions la "tradition». Celle-ci correspond à un dire pluriel analogue, du point de vue énonciatif, au "on raconte» (légousin) si fréquemment utilisé par Hérodote. Il s'agit de dires dépendant des lógoi des ancêtres (parà tôn protéron); de dires de logographes, néanmoins, susceptibles de transmettre les données les plus évidentes; de dires enfin qui se fondent sur la mémoire (mnéme $)^{27}$.

Implicitement focalisée sur Athènes, cette mémoire n'est à vrai dire pas attachée à des lieux spécifiques où elle serait l'objet d'une commémoration ritualisée. À l'écart de toute liturgie, la mémoire partagée est ici intégrée aux

26. Veyne $1971: 75-76$, repris en partie par Prost $1996: 237-262$ (256 pour la citation) dans un bon chapitre intitulé "Mise en intrigue et narrativité".

27. Thucydide 1, 9, 1-10, 1. Les lecteurs modernes de Thucydide estiment que l'historien se fonde ici notamment sur l'un des ouvrages de son contemporain Hellanicos de Lesbos : cf. FGrHist. $4 \mathrm{~F}$ 157, et Hornblower 1991 : 31-32. 
procédures de mise en discours et de configuration spatio-temporelle du passé de la communauté. En dépit du travail de rédaction écrite (suggráphein), en dépit de la visée laïque et critique qui l'anime, l'effort intellectuel de configuration mémoriale offert par Thucydide a pour produit un monument qui ressemble fort à « ce testament qu'aucune communauté humaine, sans écriture ni historiens, n'a manqué sinon de rédiger du moins de penser, où s'élabore ce mode d'emploi du passé sous forme argumentée ${ }^{28}$.

\section{Temporalités en interférence, approches convergentes}

Si la tradition peut être considérée comme une forme d'argumentation (discursive et figurative) fondée sur une mémoire culturelle partagée, anthropologues et historiens semblent être invités à partager leurs approches. La nature même de leur objet les convie à collaborer dans la saisie comparative de configurations et de représentations discursives du passé qui s'avèrent moins hétérogènes qu'on a voulu le penser. En effet, d'emblée les configurations du temps passé qui, dans les cultures exotiques comme dans la tradition occidentale, sollicitent l'intérêt des savants présentent de nombreux traits narratifs, descriptifs, argumentatifs et pragmatiques communs; ces analogies conduisent donc à une attitude comparatiste et à un échange où anthropologues et historiens sont naturellement appelés à dialoguer de manière critique. C'est en particulier le cas pour le regard que nous pouvons jeter par delà les siècles, dans une comparaison académiquement construite à travers nos propres modes d'écriture de l'histoire, sur les pratiques historiopoiétiques d'un Hérodote ou d'un Thucydide.

Mais il arrive aussi que les temporalités historiques et les espaces sociaux propres à une communauté exotique d'une part et à notre tradition culturelle de l'autre se trouvent effectivement en concurrence, invitant par nécessité le regard de l'historien à croiser celui de l'anthropologue. C'est ainsi que le système de datation et de mesure du temps européen a pu devenir le garant de l'authenticité des chroniques historiques ayant permis aux Anglais de revendiquer la dépouille mortelle du capitaine Cook, sanctifié en apothéose, tandis que dans le même temps et sur le même lieu, les récits oraux des Hawaïens contant la résurgence des fonds marins des ossements du capitaine légitimaient son intégration au temps cyclique du rite économique et politique du makakiki, rendu au dieu Lono auquel l'illustre

28. Selon la formule proposée par Gérard Lenclud (1994: 43), dans une étude qui s'efforce d'atténuer le grand partage tracé entre des sociétés de l'" oralité", attachées à une tradition fluide qui se modifierait sans cesse et les sociétés de l'écriture invitées à fixer une tradition dont elles pourraient se distancier. Pour une conception pratique et dynamique de la "mémoire culturelle » entre tradition orale et pratiques de l'écriture, voir en particulier Assmann $2000: 37-44$ et 101-123. 
voyageur anglais avait été assimilé par les indigènes ${ }^{29}$. Simultanéité et interférence spatio-temporelles forcent ici au regard comparatif.

De plus, les représentations plurielles du temps et du passé des sociétés traditionnelles et celles des sociétés occidentales peuvent s'entremêler, souvent amenées à converger en raison même de l'intégration progressive des premières au temps calendaire et biblique de la chrétienté, puis à celui de l'économie de marché ${ }^{30}$. Ainsi, à la concurrence entre représentations du temps dépendant de paradigmes culturels différents peuvent se substituer l'imbrication et l'intégration en un même contexte spatial; soit que le temps partagé par la communauté indigène débouche sur le temps politique et policé du pouvoir organisé à l'occidentale comme c'est le cas dans l'autobiographie du premier ministre de Papouasie-Nouvelle-Guinée Michael Somare (la combinaison du nom propre indigène avec un prénom chrétien à la forme anglo-saxonne est à elle seule anticipatrice et significative...) ; soit que, dans le sens en quelque sorte inverse, le retour sur le terrain papou d'un anthropologue américain a pu contribuer à un renversement millénariste des cultes rendus aux ancêtres après que le "Wasp » fut intégré dans une première phase au temps de la tradition par assimilation avec Baingap, le héros culturel de la communauté arapesh d'Ilahita; soit encore que la temporalité généalogique à la fois locale et universelle (dans l'islam) du document écrit purement fictif qui fonde l'identité de l'oasis El Ksar dans le Sud tunisien trouve une confirmation identitaire dans le temps procédural de la recherche de l'anthropologue helvéto-tunisien travaillant sur le terrain ${ }^{31}$.

Mais surtout, dans la phase de préfiguration, préalable indispensable à toute mise en discours aussi bien historiographique qu'anthropologique, praticiens du champ historique et explorateurs du terrain ethnologique subissent des contingences analogues face aux «témoignages ". Les uns et les autres se trouvent confrontés à la dialectique de la vue et de l'ouïe. En dépit de la possibilité spécifique de l'observation "participante» qui lui est offerte, le regard de l'anthropologue subit l'orientation que lui imposent, dans la distance spatiale, sa formation intellectuelle et ses propres

29. Voir, pour l'"apothéose du capitaine Cook» dont les chroniques anglaises datent la mort du 14 février 1779, les documents et récits allégués par Sahlins 1979, ainsi que 1981: 9-32, avec le commentaire de Marcus \& Fischer 1986: 103-105.

30. Différents modes de l'intégration des Blancs et de leur système économique aux temporalités papoues par l'intermédiaire du temps biblique et du récit des fils de Noé sont évoqués par Kilani, 1994 : 119-136.

31. Au sujet de la temporalité syncrétique qui traverse et organise le parcours autobiographique de Michael Somare de son village natal au palais de Port Moresby, voir mon étude de 1998 : 342-349; pour le parcours pratiquement inverse d'un anthropologue occidental, voir Tuzin 1997 : 126-156; quant à l'exemple de la reformulation de la temporalité politique musulmane au contact de l'anthropologue, il est étudié par Kilani 1992: 21-48, 95-126 et 261-317. 
préconstruits académiques. Quant à l'historien, non seulement il adopte un point de vue également orienté par son contexte académique, mais, en raison de la distance temporelle, sa perspective dépend des médiations discursives auxquelles sont immanquablement soumises les images oculaires ou la mémoire visuelle de témoins et témoignages. Dans les deux cas - l'observation directe aussi bien que l'appréhension de traces et témoignages -, la préfiguration dépend d'une rhétorique du regard qui implique, avec ses spécificités propres, une mise en forme préalable du "matériau» signifiant à réélaborer ${ }^{32}$. Historiens et anthropologues, les uns par delà le temps, les autres à travers l'espace, sont confrontés comme Hérodote à des lógoi, à des discours : déclarations et récits des informateurs indigènes d'un côté, formes verbales assumées par les documents, de l'autre, impliquant les effets schématisants, l'épaisseur polysémique et l'orientation énonciative de tout produit d'une mise en discours ${ }^{33}-$ pour l'historien et pour l'anthropologue préfigurations sans doute, mais préfigurations configurées.

Par ailleurs, praticiens de l'histoire et maîtres du terrain anthropologique se sont rejoints de manière résolue dès lors que l'anthropologie culturelle et sociale a consenti à pondérer le principe structural du travail en pure synchronie. Non seulement historiens et anthropologues se trouvent désormais confrontés à l'historicité de l'objet qu'ils construisent - et que ces derniers sont contraints de reconstruire à l'occasion de chacun de leurs retours sur le terrain -, mais parmi les représentations qui constituent la base préfigurée mais aussi déjà configurée de leurs propres procédures de mise en discours et de configuration, les différentes conceptions et représentations, souvent narratives, du passé et plus généralement du temps et de l'espace de la communauté tiennent de fait une place de choix.

Ces convergences doivent constituer pour les historiographes une invitation à s'engager dans une historía compréhensive, s'inspirant des modes d'appréhension sémiotiques de l'anthropologie culturelle et sociale, toujours prompte à insérer la conjoncture spatio-temporelle particulière dans un système de représentations relatif à une culture singulière. Mais elles les invitent aussi à exercer la nouvelle conscience critique de l'anthropologie

32. On pourra se référer, à propos de l'observation et de sa rhétorique du regard, aux réflexions d'Affergan 1987 : 137-162, et de Fabietti 1999: 33-71.

33. Autant les critiques du discours anthropologique, d'ailleurs plus aiguës dans le domaine anglosaxon qu'en France même, que les tentatives de fonder une anthropologie dialogique, faite de l'interaction entre les deux cultures que confronte par nécessité l'enquête ethnologique, ont marqué la recherche durant les trois décennies écoulées: voir le chapitre de synthèse offert par Marcus \& Fischer (1986 : 45-76) par référence aux travaux de Vincent Crapanzano, de Clifford Geertz ou de Paul Rabinow, parmi d'autres; voir aussi les indications données à ce propos par Malighetti 1998, et par Fabietti 1999 (41-46 et 232-238). 
vis-à-vis des procédures configurantes inhérentes à ses propres modes discursifs et finalement à sa rhétorique académique. Enfin, elles dessinent un engagement à confronter de manière comparative plusieurs conceptions pratiques de la temporalité, à l'intérieur d'une culture particulière, dans le cours de l'histoire d'une même communauté civilisée, ou dans le contact d'une civilisation avec d'autres systèmes et modèles culturels. Plaidoyer, donc, pour des formes d'historiographie qui soient des " historiopoiéseis " anthropologiques, des œuvres de (re-)configuration du temps et de l'espace sociaux et de leurs représentations, des récits animés par les logiques de la motivation de l'action des communautés humaines dans leurs aspects pratiques et symboliques.

\section{Mondes possibles et historicité des communautés de croyance}

Le travail de la configuration temporelle implique donc, par l'intermédiaire des procédures de la mise en discours de type anthropologique, des procédures de poiesis, de fabrication mimétique au sens où l'entend Aristote dans son Art poétique. Dans cette perspective, le monde possible ainsi créé peut en effet s'inscrire dans l'ordre de la cohérence logique impliquée par le nécessaire ou dans celui de véri-similitude morale sous-jacent au vraisemblable. C'est ainsi que les discours des historiens s'offrent désormais à nous comme des représentations configurantes de l'espace et du temps par le moyen d'opérations de sélection, de schématisation, de focalisation spatiale, de mise en séquence chronologique, de mise en intrigue et de description modélisante, de logique causale et argumentative insérant l'événement dans une conjoncture multiforme, de rhétorique discursive enfin dans un «faire-voir» qui coïncide sans doute avec les images évoquées par la mémoire individuelle et collective. À partir de ce constat, le souhait serait que l'écriture de l'histoire puisse adopter les modes d'une anthropologie soucieuse d'explorer les aspects les plus symboliques de la conjoncture, marquée qu'elle est par l'agir culturel des hommes.

Mais les discours produits par les historiens sont eux-mêmes soumis à l'historicité et au repérage spatial. Ils fabriquent des "mondes possibles » eux-mêmes pris dans le flux événementiel animé à la fois par l'écoulement du temps physique ou cosmique et par les interventions locales des hommes et de leurs communautés culturelles et sociales. En raison même de l'effet pratique déjà indiqué et inhérent à toute représentation et surtout à toute mise en discours, la vraisemblance et la cohérence du monde possible et fictionnel construit par des moyens verbaux trouvent leur validité dans une communauté de croyance particulière et dans le système des représentations qu'elle partage ; une communauté de croyance et une 
culture situées dans l'espace et dans le temps; une communauté de croyance et une tradition qui dépendent elle-mêmes de la localisation et de l'historicité culturelles.

Cette conscience d'une concomitance entre les configurations et conceptions communautaires du temps et de l'espace dans leur impact pratique et les refigurations et représentations individuelles qui en découlent dans un contexte culturel lui-même soumis à l'histoire est déjà celle bien exprimée par Maurice Halbwachs il y a presque un siècle :

"On ne peut se souvenir qu’à condition de retrouver, dans les cadres de la mémoire
collective, la place des événements passés qui nous intéressent [...]. Mais l'oubli ou la
déformation de certains de nos souvenirs s'explique aussi par le fait que ces cadres
changent d'une période à l'autre. La société, suivant les circonstances, et suivant les
temps, se représente de diverses manières le passé: elle modifie ses conventions " ${ }^{34}$.

On ajoutera que l'impact social et individuel de configurations du temps et de l'espace collectivement acceptées dans le moment de refiguration, notamment grâce au métier de mise en discours des historiens, contribue en retour à modifier ces représentations, en un mouvement à la fois dialectique et dynamique, que l'on peut souhaiter critique. Ainsi, à l'égard de la refiguration du temps qui s'opère au moment de la réception (dans mimèsis III), l'acte de mise en discours historiopoiétique et son produit configurant ne sont pas sans présenter des analogies avec l'opération de rapatriement du savoir et des pratiques culturelles indigènes que constitue en définitive la mise en discours anthropologique : intégration au-delà de la distance temporelle aux normes du savoir académique occidental parallèle à l'adaptation aux mêmes normes par-delà la distance géographique. Dans chaque cas, à travers le temps aussi bien qu'à travers l'espace, on assiste en somme à une opération de traduction transculturelle qui a en retour un impact sur les deux systèmes de représentations ainsi mis en relation ${ }^{35}$.

\section{Régimes d'historicité et logiques de temporalité}

C'est dans ce contexte d'une dialectique dynamique entre configuration et refiguration et d'une réadaptation herméneutique à des paradigmes en constante mutation que la notion de "régime d'historicité " revêt son entière pertinence. Cette catégorie semi-empirique récente a été élaborée dans une rare collaboration entre un historien et un anthropologue. Elle permet de focaliser l'attention moins sur l'immanquable inscription des

34. Halbwachs 1925 : 278-279; voir aussi Revel 2001 : 59-62, et Kilani 2003.

35. Sur le rapatriement culturel et sur l'attitude critique qu'il exige dans le sens proposé notamment par les " cultural studies ", voir par exemple les réflexions de Marcus \& Fischer 1986 : 133157 et de Kilani 1992: 311-317, et 1994: 11-26, ainsi que de Remotti 1990. Prost 1996 : 444-453 décrit fort bien les enjeux académiques du « rapatriement » du passé par les historiens. 
communautés des hommes et de leurs cultures dans le flux du temps (cosmique et social) ou sur leur manière de vivre une historicité indépendante du caractère oral ou écrit de leur tradition que sur les représentations que les hommes se font de cette inscription. Dans cette perspective, «le régime d'historicité définirait une forme culturellement délimitée, et conventionnelle, de relation au passé; l'historiographie serait l'une de ces formes et, en tant que genre, un élément symptomatique d'un régime d'historicité englobant $"{ }^{36}$. Cela signifie que la catégorie opératoire du régime d'historicité engloberait aussi bien les préfigurations communautaires et culturelles du temps que leurs configurations par le travail de facture plus spécialisé des historiopoiètes. Sans doute conviendrait-il dès lors de substituer "temporalité " à " historicité ", voire " logique " à " régime ", tant il est vrai que la pensée de l'histoire renvoie communément et de manière restrictive à la configuration du seul passé ; tant il est vrai également que le passé ne se reformule en fait qu'en tension vers le futur, à travers le hic et nunc de l'élaboration et de l'énonciation d'une manifestation symbolique, en général assumée plus ou moins collectivement en raison de sa logique interne. Et l'immanquable corrélation du nunc avec le hic nous avertit que tout régime ou logique de temporalité s'imbrique dans un "régime (ou une logique) de spatialité », localisant, pour la repérer, la configuration du temps correspondante par rapport à un espace lui aussi partagé entre écologie naturelle ou culturelle et construction symbolique.

Les possibilités de réception et de refiguration par la communauté qui les a suscitées et à laquelle elles sont destinées instituent donc les régimes et logiques de temporalité et de spatialité en «régimes de vérité ». Vérités répondant aux critères configurants du vraisemblable et de la cohérence poiétiques et fictionnels; vérités agissant sur le monde dont elles sont issues; vérités pratiques et relatives, repérées dans l'espace et dans le temps, reposant par conséquent sur des représentations et des paradigmes symboliques variant dans l'histoire et dans la géographie des cultures; mais vérités dépendant de mondes possibles en relation les uns avec les autres dans la mesure même où toute création symbolique et culturelle est fondée sur la transitivité et sur la communicabilité ${ }^{37}$. Si la notion même de « régime» implique bien l'idée de la gestion pratique d'une représentation partagée ou d'un modèle culturel, la pluralité des régimes de vérité ne saurait avoir comme corollaire une position de relativisme absolu : leur interaction et leur traductibilité l'interdisent.

36. Selon la définition donnée par Hartog \& Lenclud 1993 : 26, avec le commentaire de Hartog $2003: 17-22$ et $26-30$.

37. Entre fiabilité et suspicion, Ricœur (1998: 17) est conscient du fait que «la vérité en histoire reste ainsi en suspens, plausible, probable, contestable, bref toujours en cours de ré-écriture ". 


\section{Énonciation et régimes d'identité}

Or l'institution d'une logique de temporalité et de spatialité correspondant à un régime de vérité particulier passe par une mise en forme sémiotique qui est en général de l'ordre du discursif. Fondée sur les ressources de création fictionnelle propres à toute langue, sinon à tout langage, la configuration temporelle et spatiale se présente donc, par les procédures de mise en discours indiquées, comme une construction sémantique. Cela signifie qu'elle est régulièrement formulée en des termes polysémiques qui appellent la pluralité des lectures, la pluralité des refigurations - dans le temps et dans l'espace - suscitées par sa dimension pragmatique ${ }^{38}$.

Mais qui dit mise en discours implique une prise en charge énonciative des différents énoncés configurant temps et espace. Tout en contribuant à accentuer la polysémie du monde spatio-temporel construit dans le texte et à solliciter l'activité herméneutique et critique attachée à la lecture et à la refiguration, l'énoncé de l'énonciation organise et oriente ce monde possible à partir du point focal que représente "l'instance d'énonciation »; une instance déjà mentionnée à propos du point axial de la mise en discours narrative, avec ses coordonnées pronominales et spatio-temporelles : je, hic, nunc $^{39}$. C'est en particulier par ce biais énonciatif, dont a vu l'impact dans l'organisation narrative et discursive de la temporalité et de la spatialité, que s'affirme la dimension pragmatique de la production verbale et textuelle. Le jelnous ne se construit, dans l'espace-temps configuré dans le discours, qu'en relation avec le tu/vous qui renvoie aux actrices et aux acteurs de la lecture herméneutique et de la refiguration critique, à la fois intellectuelle et pratique. Du point de vue temporel, c'est précisément sur les procédures énonciatives que repose principalement la projection dans le futur de la construction discursive du temps passé, avec sa visée pratique.

En définitive, c'est bien une identité qui se construit autant à travers le temps et l'espace représentés et énoncés que dans le processus spatiotemporel de la mise en discours et de l'énonciation elles-mêmes. D'ordre énonciatif, cette identité est fondée sur l'entrelacs d'une représentation configurative (narrative et descriptive) du temps et de l'espace avec la

38. Rappelons que par exemple Benveniste (1974: 222-227), tout en attribuant à la fonction linguistique la double modalité de signifier (sémiotique) et de communiquer (sémantique), affirme que la polysémie "n'est que la somme institutionnalisée, si l'on peut dire, de ces valeurs contextuelles, toujours instantanées, aptes continuellement à s'enrichir, à disparaitre, bref, sans permanence, sans valeur constante".

39. Je me limite ici à réaffirmer l'indispensable distinction opératoire entre situation et processus (extradiscursif) de communication, traces textuelles (intradiscursives) de ce processus (énonciation énoncée par les moyens de l'appareil formel de l'énonciation), et énoncé lui-même (assertion, récit, description, etc.) : cf. Calame 2000a: 18-34, dans des réflexions fondées sur une abondante bibliographie; en ce qui concerne le discours anthropologique, voir aussi Affergan $1987: 213-223$. 
logique temporelle et spatiale qui organise le discours dans sa progression. Cette identité énonciative se constitue donc dans la mise en discours pour acquérir en son terme la stabilité (provisoire) qui est la condition de son institution en régime de vérité. Mais en raison même de sa nature discursive et par conséquent polysémique, cette identité configurée recèle en elle-même, dans ses paramètres temporels et spatiaux, les prémisses de nouvelles transformations. Pour le dire à nouveau avec les termes proposés par Paul Ricœur, à partir d'un noyau d'identité-idem, cette "mêmeté " d'ordre en particulier spatio-temporel se transforme en une identité-ipse; en acquérant progressivement de la consistance à travers le discours, cette "ipséité " finit par atteindre l'apparence d'une nouvelle "mêmeté »; mais en raison même de sa nature discursive, cet idem est prêt à basculer, notamment par refigurations interposées, en de nouvelles métamorphoses de l'ordre de l'ipse $e^{40}$.

Par leur caractère en général discursif et par leur forme énonciative, le régime et la logique de temporalité et de spatialité peuvent donc constituer en quelques sorte l'enveloppe d'une identité-ipse, mais d'une identité tendant à l'idem. Loin de renvoyer à un "soi » de nature psycho-philosophique, cette identité discursive, dans sa fluidité marquée par des moments de stabilité, relève du collectif et du culturel comme l'avait précisé Michel de Certeau (1975 : 49-50) :

"L'histoire se joue donc là, sur ces bords qui articulent une société avec son passé et l'acte de s'en distinguer; en ces lignes qui tracent la figure d'une actualité en la démarquant de son autre, mais qu'efface ou modifie continuellement le retour du "passé" ".

Produit symbolique, une telle identité historique est agissante de par la dimension pragmatique de toute configuration discursive; c'est ainsi qu'elle contribue à orienter le temps et l'espace du passé sur un hic et nunc dirigé vers le futur. Les représentations discursives du temps et de l'espace sont aussi, pour toute communauté culturelle, des manières de se concevoir symboliquement, de se dire et d'agir sur soi et sur les autres dans le flux du temps physique et social et dans son espace d'exercice.

Tout en retenant à titre opératoire les trois moments de la préfiguration, de la configuration et de la refiguration dans leur séquence dynamique spatio-temporelle, le renversement de perspective proposé ici tend à mieux installer l'histoire dans son statut de science humaine, à l'écart de toute phénoménologie du temps fondée en temporalité métaphysique pour dénigrer nos manières de vivre, de concevoir dans différentes formes de discours et de nous rappeler (collectivement et efficacement) notre passé,

40. Voir Ricœur 1985: 352-358, et 1990, 11-35 et 60-72, sur fond d' "identité personnelle", de cogito cartésien, mais aussi de «sujet d'énonciation». 
de manière forcément éphémère et relative, à travers le temps et l'espace organiques et physiques de notre corps propre et de son insertion environnementale.

MOTS CLÉS/KEYWORDS : configuration et représentation/configuration and representation énonciation/enunciation - espace/space - histoire et mémoire/history and memory - Paul Ricœur - temps/time.

\section{BIBLIOGRAPHIE}

Affergan, Francis

1987 Exotisme et altérité. Essai sur les fondements d'une critique de l'anthropologie. Paris, PUF.

Affergan, Francis, Silvana Borutti, Claude Calame, et al.

2003 Figures de l'humain. Les représentations de l'anthropologie. Paris, Éditions de l'EHESS.

Assmann, Jan

2000 Religion und kulturelles Gedächtnis. München, C. H. Beck.

Benveniste, Émile

1966 Problèmes de linguistique générale.

Paris, Gallimard.

1974 Problèmes de linguistique générale II. Paris, Gallimard.

\section{Borutti, Silvana}

1996 «Verità dell'evento e ruolo del soggetto nella coscienza storica " in Ettore Fagiuoli \& Marco Fortunato, eds, Soggetto e verità. La questione dell'uomo nella filosofia contemporanea. Milano, Mimesis : 235-256. 1999 Filosofia delle scienze umane. Le categorie dell'antropologia e della sociologia. Milano, Bruno Mondadori.

Bourdieu, Pierre

1982 Ce que parler veut dire. L'économie des échanges linguistiques. Paris, Fayard [nouv. éd. en 2001 : Langage et pouvoir symbolique. Paris, Seuil].

\section{Calame, Claude}

1996a Mythe et histoire dans l'Antiquité grecque. La création symbolique d'une colonie. Lausanne, Payot.

1996b Thésée et l'imaginaire athénien.

Légende et culte en Grèce antique. Lausanne, Payot [2 éd.].

1998 «Mémoire collective et temporalités en contact: Somare et Hérodote", Revue de l'histoire des religions 215 : 341-367.

2000a Le Récit en Grèce ancienne. Énonciations et représentations de poètes. Paris, Belin [ $2^{\mathrm{e}}$ éd.].

2000b Poétique des mythes dans la Grèce antique. Paris, Hachette.

2000c "Temps du récit et temps du rituel dans la poétique grecque: Bacchylide entre mythe, histoire et culte", in Catherine Darbo-Peschanski, ed., Constructions du temps dans le monde grec ancien. Paris, CNRS Éditions : 395-412.

2002 «Interprétation et traduction des cultures. Les catégories de la pensée et du discours anthropologique ", L'Homme $163:$ : 51-78.

Certeau, Michel de

1975 L'Écriture de l'histoire. Paris, Gallimard. 
Chartier, Roger

1998 Au bord de la falaise. L'histoire entre certitude et inquiétude. Paris, Albin Michel.

\section{Coquet, Jean-Claude}

1997 La Quête du sens. Le langage

en question. Paris, PUF.

\section{Darbo-Peschanski, Catherine}

1998 "L'historien grec ou le passé jugé », in Nicole Loraux \& Carles Miralles, eds, Figures de l'intellectuel en Grèce ancienne. Paris, Belin : 143-189.

Darbo-Peschanski, Catherine, ed.

2000 Constructions du temps dans le monde grec ancien. Paris, CNRS Éditions.

Edmunds, Lowell

2001 Intertextuality and the Reading of Roman Poetry. Baltimore-London, The Johns Hopkins University Press.

Fabietti, Ugo

1999 Antropologia culturale. L'esperienza e l'interpretaztone. Roma-Bari, Laterza.

Genette, Gérard

1973 Figures III. Paris, Seuil.

\section{Ginzburg, Carlo}

1989 Mythes, emblèmes, traces. Morphologie et histoire. Paris, Flammarion [éd. orig. : Miti, Emblemi, Spie. Torino, Einaudi, 1986].

Halbwachs, Maurice

1925 Les Cadres sociaux de la mémoire.

Paris, Alcan.

\section{Hartog, François}

2000 "Le témoin et l'historien", Gradhiva 27 : 1-14.

2001 [1980] Le Miroir d'Hérodote. Essai sur la représentation de l'autre. Paris, Gallimard [3 éd.].

2003 Régimes d'historicité. Présentisme et expériences du temps. Paris, Seuil.
Hartog, François \& Gérard Lenclud

1993 "Régimes d'historicité ",

in Alexandre Duku \& Norbert Dodille, eds,

L'Harmattan : 18-38.

Heidegger, Martin

1986 Etre et Temps. Paris, Gallimard [éd. orig. : Sein und Zeit. Tübingen,

Niemeyer, 1927].

Hornblower, Simon

1991 A Commentary on Thucydides I. Books I-III. Oxford, Clarendon Press.

Kilani, Mondher

1992 La Construction de la mémoire.

Le lignage et la sainteté dans l'oasis d'El Ksar. Genève, Labor \& Fides.

1994 L'Invention de l'autre. Essais sur le discours anthropologique. Lausanne, Payot.

2003 «L'art de l'oubli. Construction de la mémoire et narration historique", in Ute Heidmann, ed., Poétiques comparées des mythes. Lausanne, Payot: 212-242.

Koselleck, Rheinhardt

1990 Le futur passé: contribution à la sémantique des temps historiques. Paris, Éditions de l'EHESS [éd. orig. : Vergangene Zukunft. Zur Semantik geschichtlicher Zeiten. Frankfurt, Suhrkamp, 1979].

Lenclud, Gérard

1994 "Qu'est-ce que la tradition?", in Marcel Detienne, ed., Transcrire les mythologies. Tradition, écriture, historicité. Paris, Albin Michel : 24-44.

Malighetti, Roberto

1998 «Dal punto di vista dell'antropologo. L'etnografia del lavoro antropologico ", in Ugo Fabietti, ed., Etnografia e culture. Antropologi, informatori e politiche dell'identità. Roma, Carocci : 201-215.

Marcus, George E. \& Michael M. J. Fischer 1986 Anthropology as Cultural Critique. An Experimental Moment in the Human 
Sciences. Chicago-London, The University of Chicago Press.

Marincola, John

1997 Authority and Tradition in Ancient Historiography. Cambridge, Cambridge University Press.

Meschonnic, Henri

1990 Le Langage Heidegger. Paris, PUF.

Molet, Louis

1990 "Histoire du comput et de quelques calendriers ", in Jean Poirier, ed., Histoire des mours I.1. Les coordonnées de l'homme et la culture matérielle. Paris, Gallimard : 189-268.

Müller, Günther

1968 Morphologische Poetik. Gesammelte Aufsätze. Tübingen, Niemeyer.

Nora, Pierre

1984 «Entre Mémoire et Histoire. La problématique des lieux ", in Pierre Nora, ed., Les Lieux de mémoire. La République. Paris, Gallimard : XVII-XLII.

\section{Paty, Michel}

1996 «Sur l'histoire du problème du temps. Le temps physique et les phénomènes ", in Étienne Klein \& Michel Spiro, eds, Le Temps et sa flèche. Paris, Flammarion : 21-58.

Prost, Antoine

1996 Douze leçons sur l'histoire. Paris, Seuil.

Remotti, Francesco

1990 Noi primitivi. Lo specchio dell'antropologi. Torino, Bollati Boringhieri.
Revel, Jacques

2001 "Les sciences historiques", in Jean-Michel Berthelot, ed., Épistémologie des sciences sociales. Paris, PUF : 21-76.

Ricœur, Paul

1983 Temps et Récit. Tome I. Paris, Seuil.

1984 Temps et Récit II. La Configuration dans le récit de fiction. Paris, Seuil.

1985 Temps et Récit III. Le Temps raconté. Paris, Seuil.

1990 Soi-même comme un autre. Paris, Seuil. 1998 "La marque du passé ", Revue de Métaphysique et de Morale 1 : 7-31.

2000 La Mémoire, l'histoire, l'oubli. Paris, Seuil.

Sahlins, Marshall

1979 "L'apothéose du capitaine Cook», in Michel Izard \& Pierre Smith, eds, La Fonction symbolique. Essais d'anthropologie. Paris, Gallimard : 307-339 [repris et augmenté en 1989: Des îles dans l'histoire. Paris, Gallimard-Seuil : 114-141].

1981 Historical Metaphors and Mythical Realities. Structure in the Early History of the Sandwich Islands Kingdom. Ann Arbor, The University of Michigan Press.

\section{Tuzin, Donald}

1997 The Cassowary's Revenge. The Life and Death of Masculinity in a New Guinea Society. Chicago-London, The University of Chicago Press.

Veyne, Paul

1971 Comment on écrit l'histoire.

Essai d'épistémologie. Paris, Seuil. 
Claude Calame, Pour une anthropologie culturelle des pratiques historiographiques. - Envisagée dans une perspective discursive et énonciative, l'opération historiographique se révèle être une mise en discours du passé et de son espace. En tant que configuration et représentation, elle se fonde sur différents types de mémoire individuelle et collective qu'elle configure par différents moyens discursifs et rhétoriques. Suffisamment compréhensive, elle contribue à l'institution de régimes et de logiques de spatio-temporalité qui, eux-mêmes marqués dans le temps et dans l'espace, correspondent à des régimes de vérité pratique, ayant une efficacité discursive dans le présent. Éloignées dans l'espace et dans le temps comme c'est le cas pour la Grèce antique ou plus proches de nous, ces opérations d' "historiopoiésis" reposent sur des approches anthropologiques.
Claude Calame, For a Cultural Anthropology of Historiographical Practices. - From the discursive perspective of speech acts, the writing of history turns out to be the working out of a discourse about the past and its space. As a configuration and representation, it has grounds in various types of individual and collective memories that it configures using diverse discursive and rhetorical means. Sufficiently comprehensive, it helps institute spatiotemporal systems and "logics" that, with markers of time and space, correspond to systems of practical truth with discursive effectiveness in the present. Whether remote in space and time (as in the case of Ancient Greece) or close to us, these "historiopoiesis" operations rely on anthropological approaches. 